\title{
Four-port SNAP microresonator device
}

\author{
Manuel Crespo-Ballesteros*, Yong Yang, and Misha Sumetsky
}

Aston Institute of Photonic Technologies, Aston University, Birmingham B4 7ET, United Kingdom.

*Corresponding author: m.crespo@aston.ac.uk

Received XX Month XXXX; revised XX Month, XXXX; accepted XX Month XXXX; posted XX Month XXXX (Doc. ID XXXXX); published XX Month XXXX

It is well known from quantum mechanics that the transmission amplitude of a symmetric double-barrier structure can approach unity at the resonance condition. Similar phenomenon is observed in optics for light which propagates between two waveguides weakly coupled through a microresonator. Examples of microresonators used for this purpose include ring, photonic crystal, toroidal, and bottle microresonators. However, ring and photonic crystal photonic circuits, once fabricated, cannot be finely tuned to arrive at the mentioned resonant condition. In turn, it is challenging to predictably adjust coupling to toroidal and bottle microresonators by translating the input-output microfibers since the modes of these resonators is difficult to separate spatially. Here we experimentally demonstrate a four-port micro-device based on a SNAP microresonator introduced at the surface of an optical fiber. The eigenmodes and corresponding eigenwavelengths of this resonator are clearly identified for both polarization states by the spectrograms measured along the length of the fiber. This allows us to choose the resonant wavelength and simultaneously determine the positions of the input-output microfiber tapers to arrive at the required resonance condition.

http://dx.doi.org/10.1364/OL.99.099999

The resonant tunneling phenomenon $[1,2]$ has attracted the interest of both fundamental and applied science due to the fascinating properties of its underlying physics and its potential applications to high-speed electronics and communications. Following the pioneering work by Esaki and Tsu [3], research has mainly focused on the use of conventional semiconductors to exploit this phenomenon [4]. In particular, investigations on this topic lead to the development, among other devices, of resonant tunneling diodes, which have received a great deal of attention due to their technological applications in electronics [4].

The applications of the phenomenon of resonant tunneling have also been explored in the field of communications. As an example, resonant tunneling devices for $\mathrm{THz}$ wireless data transmission have been investigated in [5]. Another example can be found in [6] where the authors exploited the resonant tunneling effect to increase the efficiency of excitation of plasmonic nanoantennas.

Resonant tunneling devices have been investigated in optics and photonics. The simplest of these devices consists of a ring resonator coupled to two waveguides in the add-drop geometry [7]. The light from one of the waveguides can be transferred into the other one through the resonator via a resonant tunneling process. The authors of Ref. [8] designed and fabricated a photonic crystal microresonator coupled to two waveguides achieving a transmission efficiency of $89 \%$. A remarkable example is given in [9] where a power transfer efficiency of $93 \%$ has been reported using an optical four-port resonant coupler formed by a microtoroid coupled to two taper fibers. A closer example to our research can be found in [10]. The authors employed a bottle microresonator operating in add-drop configuration to obtain a transmission efficiency of $93 \%$.

The four-port resonant tunneling device proposed in this Letter is illustrated in Fig. 1. It consists of a SNAP bottle microresonator (SBMR) with nanoscale effective radius variation [11] which is evanescently coupled to the waists of two biconical microfibers oriented transversely to the SBMR axis. The two microfibers ( $\mathrm{MF}_{1}$ and $\mathrm{MF}_{2}$ ) are connected to an Optical Vector Analyzer (OVA). $\mathrm{MF}_{1}$ is used to couple light into the SBMR through port 1 and to measure the throughput light at port 2 . The signal is resonantly transmitted to $\mathrm{MF}_{2}$ through the SBMR and measured at port 3.

A SBMR device has several advantages compared to the systems mentioned above. For example, realization of the exact resonant tunneling condition for ring and photonic crystal microresonators is challenged by their insufficient fabrication precision and requires fine tuning with microheaters [12] or employing carrier injection [13]. Toroidal and bottle microresonators, on the other hand, have eigenmodes with micronscale spatial variation lengths and overpopulated resonance spectra, which complicate tuning the coupling of these resonators to the input and output waveguides. In contrast, the spectrograms of the SNAP microresonator described below show well defined and spatially separated resonant modes. This allows us to accurately select the resonant mode and determine the positions of the input-output microfibers to achieve the desired resonant tunneling condition. 


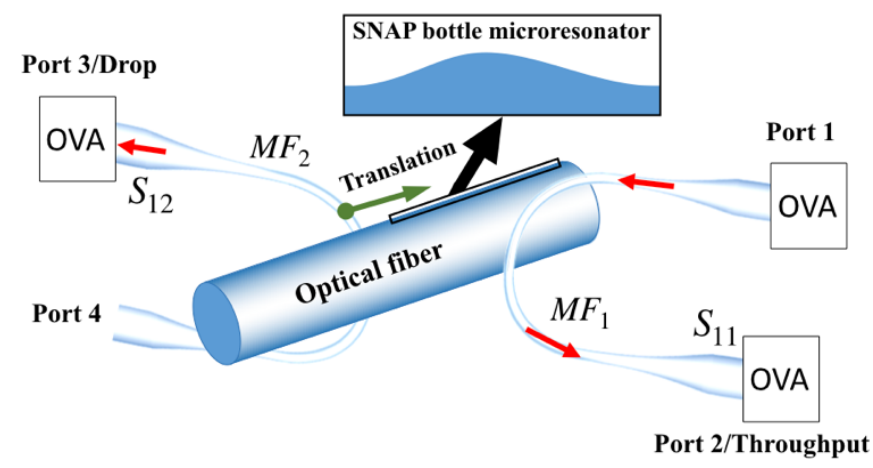

Fig. 1. Schematics of the four port resonant tunneling device proposed. The SBMR was fabricated at a silica optical fiber with $38 \mu \mathrm{m}$ radius. Two biconical microfibers are connected to OVA to measure the input and output light at the throughput and drop ports.

Light coupled into the SBMR excites Whispering Gallery Modes (WGMs) at its surface. If the WGM wavelength $\lambda$ is close to a cutoff wavelength $\lambda_{c}$ then its distribution $\Psi(\mathrm{z})$ along the SBMR axis $z$ is expressed through the Green's function of the one-dimensional wave equation [11]

$$
\begin{aligned}
& \frac{d^{2} \Psi}{d z^{2}}+\left(\beta^{2}(\lambda, z)+D_{1} \delta\left(z-z_{1}\right)+D_{2} \delta\left(z-z_{2}\right)\right) \Psi=0, \\
& \beta(\lambda, z)=\frac{4 \pi n_{0}}{\lambda_{c}}\left(\frac{\Delta r_{e f f}(z)}{r_{0}}-\frac{\lambda-\lambda_{c}-i \gamma_{c}}{\lambda_{c}}\right)^{1 / 2} .
\end{aligned}
$$

In this equation, $\beta(\lambda, z)$ is the WGM propagation constant expressed through the refractive index $n_{0}$, radius $r_{0}$ of the optical fiber, the attenuation parameter in silica $\gamma_{c}$, and effective radius variation (ERV) of the optical fiber $\Delta r_{e f f}(z)$ which determines the shape of SBMR. The positions of $\mathrm{MF}_{1}$ and $\mathrm{MF}_{2}$ at the SBMR axis enter Eq. (1) as $\mathrm{Z}_{1}$ and $\mathrm{Z}_{2}$, respectively. The effect of the SBMRmicrofiber coupling is modeled by zero-range potentials $D_{i} \delta\left(z-z_{i}\right)$, where $D_{i}, i=1,2$, are complex constants which determine the coupling between microfibers and SBMR. The shortrange approximation is justified by the fact that the axial wavelengths of the WGM are much larger than the characteristic size of the SBMR-microfiber coupling region.

The transmission amplitudes from port 1 to port 2 (throughput) and from port 1 to port 3 (drop) are expressed through the elements of the S-matrix $S_{11}$ and $S_{12}$ as, respectively,

$$
\begin{aligned}
& S_{11}(\lambda)=S_{11}^{(0)}-i C_{1} C_{1}^{*} G\left(\lambda, z_{1}, z_{1}\right), \\
& S_{12}(\lambda)=S_{12}^{(0)}-i C_{1} C_{2}^{*} G\left(\lambda, z_{1}, z_{2}\right),
\end{aligned}
$$

where $G\left(\lambda, z_{1}, z_{2}\right)$ is the Green's function of Eq. (1). The terms $S_{11}^{(0)}$ and $S_{12}^{(0)}$ represent the non-resonant components of the corresponding transmission amplitudes. Constants $C_{1}$ and $C_{2}$ are the microfibers-SBMR coupling parameters [11].

In the resonance approximation, when wavelength $\lambda$ is close to wavelength $\lambda_{n}$ of one of WGMs of the microresonator, the drop transmission amplitude

$$
S_{12}\left(\lambda, z_{1}, z_{2}\right)=S_{12}^{(0)}-\frac{i C_{1} C_{2}^{*} \Psi_{n}\left(z_{1}\right) \Psi_{n}\left(z_{2}\right)}{\kappa\left(\lambda-\lambda_{n}\right)+\Delta_{n}+i\left(\kappa \gamma_{c}+\Sigma_{n, 1}+\Sigma_{n, 2}\right)},
$$

where $\Psi_{n}(z)$ is the axial distribution of this mode determined by Eq. (1) for the isolated SBMR, i.e. for $D_{1}=D_{2}=0$, and $\kappa=\left(8 \pi^{2} n_{0}^{2}\right)^{-1} \lambda_{c}^{3}$. The term $\Delta_{n}=\operatorname{Re}\left\{D_{1}\right\}\left|\Psi_{n}\left(z_{1}\right)\right|^{2}+\operatorname{Re}\left\{D_{2}\right\}\left|\Psi_{n}\left(z_{2}\right)\right|^{2}$ accounts for the shift of resonant wavelength $\lambda_{n}$ due to coupling with microfibers. Finally, the terms $\Sigma_{n, i}=\operatorname{Im}\left\{D_{i}\right\}\left|\Psi_{n}\left(z_{i}\right)\right|^{2}$ determine the radiation losses through the microfibers.

Several conditions are required to achieve the resonance transmission power $\left|S_{12}\left(\lambda, z_{1}, z_{2}\right)\right|^{2}$ between microfibers $\mathrm{MF}_{1}$ and $\mathrm{MF}_{2}$ equal to unity. First, both fibers should operate in the strong overcoupling regime, $\Sigma_{n, i} \gg \kappa \gamma_{c}$, so that the intrinsic losses determined by $\gamma_{c}$ can be neglected. Next, the condition of lossless coupling, $\operatorname{Im}\left\{D_{i}\right\}=\frac{1}{2}\left|C_{i}\right|^{2}$, should be required (notice that generally $\operatorname{Im}\left\{D_{i}\right\} \geq \frac{1}{2}\left|C_{i}\right|^{2}$ ) [11]. Finally, the resonant transmission should give the major contribution into $S_{12}\left(\lambda, z_{1}, z_{2}\right)$, i.e., the nonresonant transmission amplitude should be negligible, $\left|S_{12}^{(0)}\right| \ll 1$. If all these three conditions are satisfied then Eq. (3) yields

$$
\begin{aligned}
& \left|S_{12}\left(\lambda, z_{1}, z_{2}\right)\right|^{2} \\
& =\frac{4\left|C_{1}\right|^{2}\left|C_{2}\right|^{2}\left|\Psi_{n}\left(z_{1}\right)\right|^{2}\left|\Psi_{n}\left(z_{2}\right)\right|^{2}}{4\left[\kappa\left(\lambda-\lambda_{n}\right)+\Delta_{n}\right]^{2}+\left(\left|C_{1}\right|^{2}\left|\Psi_{n}\left(z_{1}\right)\right|^{2}+\left|C_{2}\right|^{2}\left|\Psi_{n}\left(z_{2}\right)\right|^{2}\right)^{2}}
\end{aligned}
$$
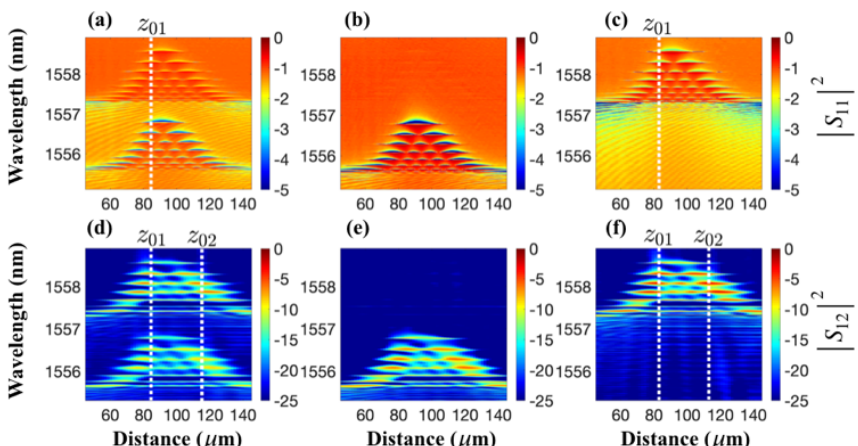

Fig. 2. (a) Spectrogram of the resonant transmission power at the throughput port $\left|S_{11}\right|^{2}$. After diagonalizing $J_{\text {SBMR }}(\lambda)$, the two polarizations are split in (b) and (c). The spectrogram in (d) corresponds to the transmission power at the drop port $\left|S_{12}\right|^{2}$. The transmission power of two separated polarizations are plotted separately in (e) and (f).

From this equation, the drop power $\left|S_{12}\right|^{2}$ reaches unity at the resonant wavelength $\lambda_{r n}=\lambda_{n}-\kappa^{-1} \Delta_{n}$ if

$$
C_{1} \Psi_{n}\left(z_{1}\right)=C_{2} \Psi_{n}\left(z_{2}\right) \text {. }
$$

This condition implies that there is a variety of mutual positions of the microfibers that result in complete resonant transmission. 
Notice that the SBMR does not need to be symmetric to satisfy this condition which makes the proposed device rather flexible to be appropriately tuned by moving microfibers along the SBMR and their own axes.

Similar to other types of optical microresonators [14-16], resonant propagation through SBMR strongly depends on the polarization of light. Commonly, control of the polarization is performed by its tuning at the input channel with a polarization controller [10,17-19]. Alternatively, in our experiments, the polarization states of SBMR were separated numerically. It is reasonable to assume that the fibers connecting the OVA to our device are lossless, so the Jones matrices describing the propagation along these fibers are unitary within a constant scalar factor. Then, within a constant factor, the measured Jones matrix $J_{\text {meas }}(\lambda)$ can be expressed through the diagonalized Jones matrix of the SBMR $J_{S B M R}(\lambda)$ (transforming its TE and TM polarization states) as $J_{\text {meas }}(\lambda)=U_{\text {out }} J_{S B M R}(\lambda) U_{\text {in }} \quad$ where $U_{\text {in }}$ and $U_{\text {out }}$ are $2 \times 2$ unitary matrices that account for the field transformation in the input and output of the OVA, optical fibers, as well as the unitary transformation of the SBMR to the diagonal form representing the transformation of its polarization states. Since $U_{\text {in }}$ and $U_{\text {out }}$ are unitary matrices, we can rearrange the latter equation to obtain $J_{S B M R}(\lambda)=U_{\text {out }}^{\dagger} J_{\text {meas }}(\lambda) U_{\text {in }}^{\dagger}$. Unitary matrices $U_{\text {in }}^{\dagger}$ and $U_{\text {out }}^{\dagger}$ contain seven free parameters which are determined by minimizing the off-diagonal elements of $J_{S B M R}(\lambda)$. The diagonal elements of the determined $J_{S B M R}(\lambda)$ are the transmission amplitude for each polarization state (TE or TM). We naturally assume that, during a single scan over the bandwidth of $5 \mathrm{~nm}$ performed in our experiments, these parameters do not change with wavelength noticeably. Furthermore, these parameters remain constant over the whole duration of the experiment, which includes recording of multiple spectrograms, if the physical properties of the input-output fibers and environment remain unaltered.

In our experiment, the SBMR was fabricated by nanoscale ERV of a $38 \mu$ m diameter optical fiber by local annealing with a $\mathrm{CO}_{2}$ laser $[20,11]$. Microfibers $\mathrm{MF}_{1}$ and $\mathrm{MF}_{2}$ were fabricated in a ceramic microheater by pulling a standard optical fiber to arrive at a waist diameter of approximately $1.5 \mu \mathrm{m}$. Subsequently, the microfibers were U-shaped as illustrated in Fig. 1 and mounted on a 3D linear stages, which were used to move them along the SBMR and periodically touch it to measure the transmission amplitudes.

First, the SBMR was characterized by measuring the resonant transmission power $\left|S_{11}\left(\lambda, z_{1}\right)\right|^{2}$ at the throughput port as a function of wavelength $\lambda$ and $\mathrm{MF}_{1}$ position $z_{1}$. The scanning resolution was $1 \mu \mathrm{m}$ in $z_{1}$ and $1.25 \mathrm{pm}$ in $\lambda$. In this configuration, the second microfiber $\mathrm{MF}_{2}$ was not in contact with the SBMR and had no effect on the measurements. The measured spectrogram $\left|S_{11}\left(\lambda, z_{1}\right)\right|^{2}$ is shown in Fig. 2(a). Remarkably, two series of resonances in this spectrogram correspond to two different polarizations. After diagonalizing $J_{S B M R}(\lambda)$, these two polarizations (named SOP 1 and SOP 2) are uncoupled as shown in the spectrograms of Figs. 2(b) and (c). Notice that the spectrograms of our SBMR are not overcrowded and have well separated resonances because of the nanoscale ERV of the SBMR and a relatively small radius of the fiber used for its fabrication. In each series, we clearly see the fundamental axial mode, $\Psi_{1}(z)$, followed by the second axial mode, $\Psi_{2}(z)$, and next axial modes, $\Psi_{3}(z), \ldots, \Psi_{8}(z)$.

The maximum detected transmission amplitude $S_{12}$ for our device is determined as follows. First, in order to arrive at the condition of strong overcoupling and relatively small intrinsic losses, $\Sigma_{n, i} \gg \kappa \gamma_{c}$, we placed $\mathrm{MF}_{1}$ at $z_{1}=z_{01}$, which corresponds to the maximum value of $\left|\Psi_{2}(z)\right|^{2}$ indicated by dashed vertical line in Fig. 2(a). The resonant wavelengths of this mode for each state of polarization are $\lambda_{2}^{+}=1556.54 \mu \mathrm{m}$ and $\lambda_{2}^{-}=1558.32 \mu \mathrm{m}$ ,where the plus and minus signs correspond to SOP 1 and SOP 2 respectively. Fig. 3(a) depicts the throughput power for each polarization after the diagonalization of $J_{S B M R}(\lambda)$ at $z_{01}$ together with the throughput power directly measured by the OVA.

Next, the transmission amplitude at the drop port, $S_{12}\left(\lambda, z_{01}, z_{2}\right)$, was measured for the fixed position of $\mathrm{MF}_{1}$ at $z_{1}=z_{01}$ and variable $z_{2}$. In this case, the scanning resolution of $\mathrm{MF}_{2}$ position $z_{2}$ was $1.5 \mu \mathrm{m}$ while the wavelength resolution was 1.25 pm. Fig. 2(d) shows the spectrogram of the directly measured transmission power $\left|S_{12}\left(\lambda, z_{01}, z_{2}\right)\right|^{2}$ and Figs. 2(f) and (e) show the transmission power for separated SOP 1 and SOP 2 . The maximum transmission for the 2nd axial resonant mode $\Psi_{2}(z)$ was measured at $z_{2}=z_{02}$, indicated in Fig. 2(d) and Fig. 2(f) which is nearly but not totally symmetric with respect to $z_{01}$.

The drop power for both polarizations measured at $z_{2}=z_{02}$ is shown in Fig. 3(b). At $\lambda_{2}^{+}$and $\lambda_{2}^{-}$, the measurements returned a value of the drop power of $\left|S_{12}\left(\lambda_{2}^{+}, z_{01}, z_{02}\right)\right|^{2}=0.271$ for SOP 1 and $\left|S_{12}\left(\lambda_{2}^{-}, z_{01}, z_{02}\right)\right|^{2}=0.636$ in the case of SOP 2 .

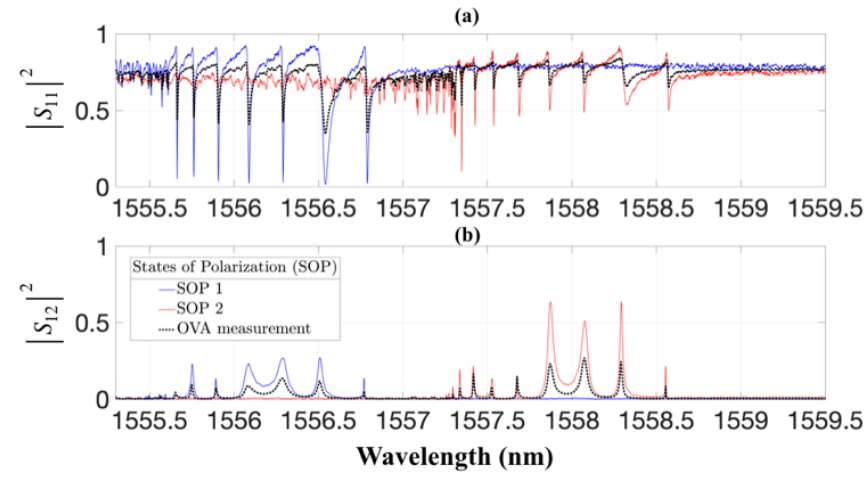

Fig. 3. (a) Spectrum of the throughput power $\left|S_{11}\right|^{2}$ measured at $\mathrm{z}_{1}=\mathrm{z}_{01}$. The two polarization states have been uncoupled after diagonalizing $J_{\text {SBMR }}(\lambda)$. (b) Drop power $\left|S_{12}\right|^{2}$ measured at $\mathrm{z}_{2}=\mathrm{Z}_{02}$ for the two states of polarization.

Finally, Fig. 4 shows the comparison of experimental results for the SOP 2 with theoretical modelling based on Eq. (1). In our theoretical simulation, we optimized the ERV $\Delta r_{\text {eff }}(z)$ and coupling 
parameters $C_{i}$ and $D_{i}$ to fit the experimental data shown in Fig. 2(c) and (f). We introduced an asymmetric distribution of the effective radius $\Delta r_{\text {eff }}(z)$ which allowed us to replicate the experimental spectrogram shown in Fig. 2. We found that our experimental spectrograms are well matched by theory if $\left|C_{1}\right|^{2}=\left|C_{2}\right|^{2}=0.02 \mu m^{-1}$ and $D_{1}=D_{2}=0.004+0.0133 i \mu m^{-1}$. The non-resonant components of the throughput and drop powers were set to $S_{11}^{(0)}=0.687-0.569 i$ and $S_{12}^{(0)}=0.035$. In contrast to the ideal transmission situation where $\operatorname{Im}\left\{D_{i}\right\}=\frac{1}{2}\left|C_{i}\right|^{2}$, the value of the imaginary part of $D_{i}$ was noticeably larger and corresponded to $\operatorname{Im}\left\{D_{i}\right\}=\frac{2}{3}\left|C_{i}\right|^{2}$.
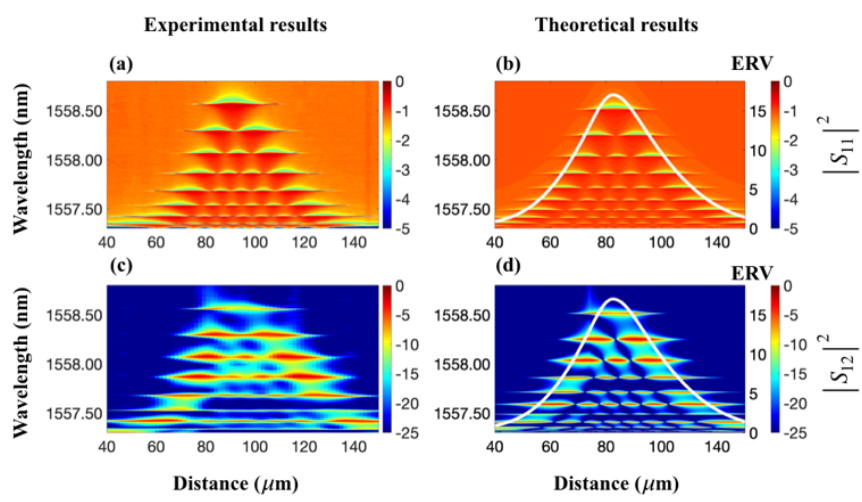

Fig. 4. Spectrograms (a) and (c) represent the experimental measurements of $\left|S_{11}\right|^{2}$ and $\left|S_{12}\right|^{2}$ from Fig. 2(c) and (f), respectively. The spectrograms (b) and (d) are the corresponding theoretical spectrograms SBMR which profile is depicted as the white line in the figures.

In conclusion, we have demonstrated a four-port resonant tunneling device fabricated of a SNAP microresonator coupled to two input-output microfibers. A bottle-shaped microresonator with characteristic effective radius variation of $17 \mathrm{~nm}$ and characteristic axial variation length of WGMs $\sim 10 \mu \mathrm{m}$ was fabricated at the surface of a $38 \mu \mathrm{m}$ diameter fiber. The large characteristic axial variation length of the WGMs allowed us to accurately position the microfibers in order to arrive at the exact resonance condition. The resonant tunneling transmission efficiency between the two microfibers was 0.635 . The value achieved in our experiment was limited by the attenuation of WGMs due to the scattering losses at the microfiber-microresonator contact regions as well as due to the material losses and surface roughness of the fiber. Scattering at the contact regions, which we believe gave the major contribution to the WGM attenuation, can be reduced if the microfibers are placed at a nanometer scale distance from the microresonator $[7,8]$. In our experiment, the transmission and spectral properties of the fabricated SNAP microresonator were not specially designed and the simplest bottle-shape SNAP microresonator was considered. In the future, more complex microresonator structures with four or more ports, e.g., series of coupled microresonators, can be designed and fabricated based on the advanced SNAP technology [21, 22]. We suggest that these new optical resonant micro-devices will find applications in classical and quantum signal processing and sensing systems.
Funding. Royal Society Wolfson Research Merit Award (WM130110); Engineering and Physical Sciences Research Council (EPSRC) (grant EP/P006183/1); Horizon 2020 MCSA-COFOUND MULTIPLY (H2020 GA 713694).

Acknowledgment. We thank Sajid Zaki for help in theoretical modelling.

\section{References}

1. D. Bohm, Quantum Theory (Dover Publications, inc., New York, New York, NY, USA, 1951).

2. B. Ricco and M. Y. Azbel, Phys. Rev. B 291970 (1984).

3. L. Esaki and R. Tsu, IBM J. Res. Dev. 14, 61 (1970).

4. H. Mizuta and T. Tanoue, The Physics and Applications of Resonant Tunnelling Diodes (Cambridge University Press, New York, NY, USA 2010).

5. N. Oshima, K. Hashimoto, S. Suzuki, and M. Asada, Electron. Lett. 52, 1897 (2016).

6. A. V. Uskov, J. B. Khurgin, I. E. Protsenko, I. V. Smetanin, and A. Bouheler, Nanoscale. 8, 14573 (2016).

7. W. Bogaerts, P. de Heyn, T. van Vaerenbergh, K. de Vos, S. Kumar Selvaraja, T. Claes, P. Dumon, P. Bienstman, D. van Thourhout, and R. Baets, Laser Photonics Rev. 6, 47 (2012).

8. M. Notomi, A. Shinya, S. Mitsugi, E. Kuramochi, and H.-Y. Ryu, Opt. Express 12, 1551 (2004).

9. H. Rokhsari and K. J. Vahala, Phys. Rev. Lett. 92, 253905 (2004).

10. M. Pöllinger and A. Rauschenbeutel, Opt. Express 18, 17764 (2010).

11. M. Sumetsky, Opt. Express 20, 22537 (2012).

12. F. Morichetti, C. Ferrari, A. Canciamilla, and A. Melloni, Laser Photonics Rev. 6, 74 (2012).

13. T. A. Ibrahim, W. Cao, Y. Kim, J. Li, J. Goldhar, P. . Ho, and C. H. Lee, IEEE Photonics Technol. Lett. 15, 36 (2003).

14. G. Guan and F. Vollmer, Appl. Phys. Lett. 86, 121115 (2005).

15. P. Bianucci, C. R. Fietz, J. W. Robertson, G. Shvets, and C.-K. Shih, Opt. Lett. 32, 2224 (2007).

16. C. Junge, D. O'Shea, J. Volz, and A. Rauschenbeutel, Phys. Rev. Lett. 110, 213604 (2013).

17. M. Chin, Opt. Express 11, 1724 (2010).

18. F. Moni, J. Friedlein, S. K. Ozdemir, and L. Yang, J. Lightwave Technol. 30, 3306 (2012).

19. M. Mohd Nasir, S. Bakhtiari Gorajoobi, G. Senthil Murugan, and M. Zervas, J. Opt. Soc. Am. B 36, 705 (2019).

20. N. A. Toropov and M. Sumetsky, Opt. Lett. 41, 2278 (2016).

21. F. Shen, X. Shu, L. Zhang, and M. Sumetsky, Opt. Lett. 41, 2795 (2016)

22. D.I L. P. Vitullo, S. Zaki, G. Gardosi, B. J. Mangan, R. S. Windeler, M. Brodsky, and M. Sumetsky, Opt. Lett. 43, 4316 (2018). 


\section{References with titles}

1. D. Bohm, Quantum Theory (Dover Publications, Inc., New York, 1951).

2. B. Ricco and M. Y. Azbel, "Physics of resonant tunneling. The onedimensional double-barrier case," Phys. Rev. B 29, 1970 (1984).

3. L. Esaki and R. Tsu, "Superlattice and Negative Differential Conductivity in Semiconductors," IBM J. Res. Dev. 14, 61 (1970).

4. H. Mizuta and T. Tanoue, The Physics and Applications of Resonant Tunnelling Diodes (Cambridge University Press, 2010).

5. N. Oshima, K. Hashimoto, S. Suzuki, and M. Asada, "Wireless data transmission of $34 \mathrm{Gbit} / \mathrm{s}$ at a $500-\mathrm{GHz}$ range using resonanttunnelling- diode terahertz oscillator," Electron. Lett. 52, 1897 (2016).

6. A. V. Uskov, J. B. Khurgin, I. E. Protsenko, I. V. Smetanin, and A. Bouhelier, "Excitation of plasmonic nanoantennas by nonresonant and resonant electron tunnelling," Nanoscale 8, 14573 (2016).

7. W. Bogaerts, P. de Heyn, T. van Vaerenbergh, K. de Vos, S. Kumar Selvaraja, T. Claes, P. Dumon, P. Bienstman, D. van Thourhout, and R. Baets, "Silicon microring resonators," Laser Photonics Rev. 6, 47 (2012).

8. M. Notomi, A. Shinya, S. Mitsugi, E. Kuramochi, and H.-Y. Ryu, "Waveguides, resonators and their coupled elements in photonic crystal slabs," Opt. Express 12, 1551 (2004).

9. H. Rokhsari and K. J. Vahala, "Ultralow loss, high Q, four port resonant couplers for quantum optics and photonics," Phys. Rev. Lett. 92, 253905 (2004).

10. M. Pöllinger and A. Rauschenbeutel, "All-optical signal processing at ultra-low powers in bottle microresonators using the Kerr effect," Opt. Express 18, 17764 (2010).

11. M. Sumetsky, "Theory of SNAP devices: basic equations and comparison with the experiment," Opt. Express 20, 22537 (2012).

12. F. Morichetti, C. Ferrari, A. Canciamilla, and A. Melloni, "The first decade of coupled resonator optical waveguides: Bringing slow light to applications," Laser Photonics Rev. 6, 74 (2012).

13. T. A. Ibrahim, W. Cao, Y. Kim, J. Li, J. Goldhar, P.-. Ho, and C. H. Lee, "All-optical switching in a laterally coupled microring resonator by carrier injection," IEEE Photonics Technol. Lett. 15, 36 (2003).

14. G. Guan and F. Vollmer, "Polarized transmission spectra of the fibermicrosphere system," Appl. Phys. Lett. 86, 121115 (2005).

15. P. Bianucci, C. R. Fietz, J. W. Robertson, G. Shvets, and C.-K. Shih, "Whispering gallery mode microresonators as polarization converters," Opt. Lett. 32, 2224 (2007).

16. C. Junge, D. O'Shea, J. Volz, and A. Rauschenbeutel, "Strong Coupling between Single Atoms and Nontransversal Photons," Phys. Rev. Lett. 110, 213604 (2013).

17. M. Chin, "Polarization dependence in waveguide-coupled microresonators," Opt. Express 11, 1724 (2010).

18. F. Moni, J. Friedlein, S. K. Ozdemir, and L. Yang, "A Robust and Tunable Add - Drop Filter Using Whispering Gallery Mode Microtoroid Resonator," J. Lightwave Technol. 30, 3306 (2012).

19. M. Mohd Nasir, S. Bakhtiari Gorajoobi, G. Senthil Murugan, and M. Zervas, "Polarization Effects in Optical Fibers," J. Opt. Soc. Am. B 36, 705 (2019).

20. N. A. Toropov and M. Sumetsky, "Permanent matching of coupled optical bottle resonators with better than $016 \mathrm{GHz}$ precision," Opt. Lett. 41, 2278 (2016).

21. F. Shen, X. Shu, L. Zhang, and M. Sumetsky, "Fabrication of surface nanoscale axial photonics structures with a femtosecond laser," Opt. Lett. 41, 2795 (2016)

22. D.I L. P. Vitullo, S. Zaki, G. Gardosi, B. J. Mangan, R. S. Windeler, M. Brodsky, and M. Sumetsky, "Tunable SNAP microresonators via internal ohmic heating," Opt. Lett. 43, 4316 (2018). 